\title{
Chain Dimensions of a Polymer Confined between Two Plates: a Mean Field Theoretical Approach
}

\author{
Kohzoh SHIOKAWA \\ Department of Applied Science, Faculty of Engineering, \\ Kyushu University, Hakozaki, Higashi-ku, \\ Fukuoka 812, Japan
}

(Received September 20, 1990)

\begin{abstract}
The chain dimensions of a polymer confined between two plates in dilute and in semi-dilute regimes were studied in the presence of the excluded volume interaction using the mean field theory as originated by Flory. The component of mean dimension perpendicular to the plates, $R_{z}$ approaches rapidly the plate distance, $D$ with decreasing $D / R_{z \infty}$, and with increasing expansion factor, $\alpha_{\infty}$, where the suffix $\infty$ denotes the value of an unconfined chain. Simple relations for the parallel component of the linear expansion factor, $\alpha_{x}$ are derived in the $D / R_{z \infty} \rightarrow 0$ limit as $\alpha_{x}^{4}-\alpha_{x}^{2}=\left(\alpha_{\infty}^{4}-\alpha_{\infty}^{2}\right)\left(R_{z \infty} / D\right)$ in a dilute regime, and as $\alpha_{c x}^{6}-\alpha_{c x}^{4}=\left(\alpha_{\infty}^{6}-\alpha_{\infty}^{4}\right)\left(R_{z \infty} / D\right)^{2}\left(C_{\infty}^{*} / C\right)$ in a semi-dilute regime, where $C$ and $C^{*}$ are the concentration of segment and the crossover concentration, respectively. The former is consistent with the scaling prediction by Daoud and deGennes; however, the latter is inconsistent with it. A plate distance-concentration diagram composed of 4 regimes, of a confined polymer solution is proposed.

KEY WORDS Chain Dimension / Confined Polymer / Slit / Slab / Excluded

Volume / Mean Field Theory / Dilute / Semi Dilute /
\end{abstract}

The chain dimensions of a polymer confined between two plates are interesting not only from a theoretical point of view, but also because of its role in many practical applications, such as the steric stabilization of colloidal dispersions. The chain dimensions of a polymer with the excluded volume interaction, confined between two plates separated by a distance $D$, have been investigated theoretically by some authors.

Daoud and deGennes ${ }^{1}$ studied this problem using the scaling theory and the blob concept. They predicted that the chain dimensions increase proportionally $D^{-1 / 4}$ with decreasing D.

Wang, Nemirovsky and Freed ${ }^{2}$ investigated this problem using the $\varepsilon$ expansion method. They obtained the explicit form of the component of the mean square end-to-end distance parallel to the plates, $\left\langle R_{x}^{2}\right\rangle$ assuming the chain dimensions to be much smaller than $D$.

The present author $^{3}$ derived the full crossover dependence of the end-to-end distance of a confined chain on $D$, using the homotopy parameter expansion method as proposed by Oono. ${ }^{4}$ It was found that the reduced value of the perpendicular component of the mean square end-to-end distance of a perturbed chain, $\left\langle R_{z}^{2}\right\rangle /\left\langle R_{z}^{2}\right\rangle_{\infty}$ is almost the same as that of an unperturbed chain, where $\infty$ denotes the value of an unconfined chain. The parallel component, $\left\langle R_{x}^{2}\right\rangle /\left\langle R_{x}^{2}\right\rangle_{\infty}$ is expressed as $\left\langle R_{x}^{2}\right\rangle \mid\left\langle R_{x}^{2}\right\rangle_{\infty}=1+c\left\langle R_{z}^{2}\right\rangle_{\infty}^{1 / 2} / D$, where $c$ is a quantity slightly dependent on $D /\left\langle R_{z}^{2}\right\rangle_{\infty}^{1 / 2}$.

These theories predict the chain dimensions in the scaling limit, i.e., the suitable long chain in a good solvent. The crossover behavior between the chain dimensions in a marginal 
solvent and that in a good solvent have not yet been predicted.

The concentration dependence of the dimensions of an unconfined chain has been studied by many authors. ${ }^{5-8}$ These studies predict that the excluded volume interaction is screened by penetrating segments in the semi-dilute regime. The chain dimensions approach the unperturbed ones when the concentration, $C$ increases. The concentration dependence of the dimensions of the confined chain has been reported only by Daoud and deGennes ${ }^{1}$ using the scaling argument. They assumed that the chain with the excluded volume interaction behaves as an unperturbed chain in a semi-dilute regime even in the case where the chain is confined in a thin slit. They predicted the chain dimensions, $R$ are proportional to $N^{1 / 2} D^{-1} C^{-1}$, where $N$ is the polymerization index.

The mean field theory originated by Flory provides the correct exponents in the relation between $R$ and $N$, not only in 3 dimensional space but also in other dimensional spaces. ${ }^{9}$ Turban ${ }^{10}$ reported that the mean fields theory gave the same exponent as predicted by the scaling argument, not only for $N$ but also for $D$ for a confined chain in the case of $D \ll R$, in the dilute regime, as already pointed out by Daoud and deGennes. ${ }^{1}$

In this paper, we studied the full crossover dependence of the chain dimensions on the plate distance and on the strength of the excluded volume interaction in dilute and semi-dilute regimes using the mean field theory as originated by Flory.

\section{MODEL}

A model chain consists of $N$ free rotating bonds of the unit length. The $z$ axis is taken perpendicular to the plates and the $x$ and $y$ axes are taken parallel to the plates.

The distribution function for the end-to-end vector $\boldsymbol{R}$ of an unperturbed chain with $N$ bonds is expressed as ${ }^{3}$
$G_{0}(\boldsymbol{R}, N)=$
$\left(\frac{3}{2 \pi N}\right) \exp \left(-\frac{3\left(R_{x}^{2}+R_{y}^{2}\right)}{2 N}\right) E\left(\frac{N}{6 D^{2}}, \frac{R_{z}}{D}\right) \frac{1}{D}$

The suffixes $x$ and $y$ denote the components parallel to the plates and $z$ denotes the perpendicular one. $E(x, y)$ is given as

$$
\begin{aligned}
& E(x, y)= \\
& \frac{\sum_{k}[(1-y) \cos (k \pi y)+\sin (k \pi y) / k \pi] \exp \left(-k^{2} \pi^{2} x\right)}{\sum_{k}^{\prime}\left(8 / k^{2} \pi^{2}\right) \exp \left(-k^{2} \pi^{2} x\right)}
\end{aligned}
$$

where $\sum_{k}^{\prime}$ denotes the sum over odd values of $k$.

The distribution function for the end-to-end distance of a perturbed chain is assumed to be

$$
G(\boldsymbol{R}, N, C)=G_{0}(\boldsymbol{R}, N) \exp \left(-v Z_{2}(\boldsymbol{R}, C)\right)
$$

where $Z_{2}$ is the number of two body contacts between segments, and $v$ is the excluded volume of a segment.

$Z_{2}$ can be evaluated by the integration of the squared segment density over the chain volume in a dilute regime. However, it is difficult to estimate the segment density as a function of $\boldsymbol{R}$. We assume that segments are distributed uniformly in the volume occupied by the chain, $V$. We get $Z_{2}=(N / V)^{2} V$ in a dilute regime.

The chain volume is evaluated using the equivalent ellipsoid, whose principal axes are proportional to $R_{x}, R_{y}$ and $R_{z}$. Then we get $V \simeq R_{x} R_{y} R_{z}$. We get the distribution function of a perturbed chain in a dilute regime as

$$
G(\boldsymbol{R}, N)=G_{0}(\boldsymbol{R}, N) \exp \left(-v c_{2}^{\prime} N^{2} / R_{x} R_{y} R_{z}\right)
$$

where $c_{2}^{\prime}$ is a numerical constant.

The expansion of chain dimensions reduces the number of segment contacts in a dilute regime, because the segment density in a chain decreases with increasing chain dimensions. In a semi-dilute regime, the expansion of chain dimensions augments the number of segment contacts with segments belonging to surrounding chains, because polymer chains overlap 
each other. The total number of segment contacts does not decrease with chain expansion in a semi-dilute regime in contrast to a dilute regime. This means that some of the segment contacts are ineffective for the chain expansion.

We assume that the segments are distributed uniformly in an equivalent ellipsoid. Furthermore, we assume that the fraction of segment contacts effective for the chain expansion is proportional to the ratio of the number of segments belonging to the chain $(=N)$, and to the number of segments contained in the chain volume $(=C V){ }^{7}$ Then we get $Z_{2}=$ $(N / V)^{2} V(N / C V)$ in the semi-dilute regime.

Finally we get the distribution function of a perturbed chain as

$$
G(\boldsymbol{R}, N)=G_{0}(\boldsymbol{R}, N) \exp \left(-v c_{2}^{\prime \prime} N^{3} / R_{x}^{2} R_{y}^{2} R_{z}^{2} C\right)
$$

for a semi-dilute regime, where $c_{2}^{\prime \prime}$ is a numerical constant.

\section{MEAN DIMENSION}

\section{Dilute Regime}

It is impossible to calculate analytically the mean square values of $R_{x}, R_{y}$ and $R_{z}$ using eq 4. The roots of the mean square of $R_{x}, R_{y}$ and $R_{z}$ are evaluated approximately following the procedure by Hermans and Overbeek. ${ }^{11}$ The approximate mean value of $x$ weighted with $G(x)$ is equal to the value at which the function $x G(x)$ has a maximum. In other words, $x G(x)$ is regarded as a delta function. The maximizing condition is

$$
\begin{aligned}
& \frac{\partial}{\partial R_{x}}\left(\ln R_{x}+\ln G_{0}-c_{2}^{\prime} v N^{2} / R_{x} R_{y} R_{z}\right) \\
& =1 / R_{x}-3 R_{x} / N+c_{2}^{\prime} v N^{2} / R_{x}^{2} R_{y} R_{z}=0
\end{aligned}
$$

The parallel component of linear expansion factor, $\alpha_{x}=R_{x} /(N / 3)^{1 / 2}$ (henceforth $R_{x}, R_{y}$ and $R_{z}$ denote the mean values) is given as

$$
\alpha_{x}^{2}-1=c z / \alpha_{x} \alpha_{y} \alpha_{z}
$$

where $c$ is a numerical constant, and $z$ is the excluded volume parameter defined as $(3 / 2 \pi)^{3 / 2} v N^{1 / 2}$. In the same manner, the following equations are derived.

$$
\begin{gathered}
\alpha_{y}^{2}-1=c z / \alpha_{x} \alpha_{y} \alpha_{z} \\
F\left(N / 6 D^{2}, R_{z} / D\right)-1=c z / \alpha_{x} \alpha_{y} \alpha_{z}
\end{gathered}
$$

where $F(x, y)$ is defined as

$F(x, y)=$

$(1-y)\left[\sum_{k} k \pi y \sin (k \pi y) \exp \left(-k^{2} \pi^{2} x\right)\right]$

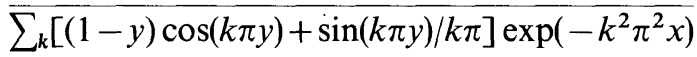

$F(x, y)$ can be approximated as

$F(x, y)= \begin{cases}y^{2} / 2 x & x \rightarrow 0 \\ 0.906 \pi^{2} y^{2} & y \simeq 0.33, x>0.16 \\ 3 y /(1-y) & y \rightarrow 1, x>0.16\end{cases}$

$F(x, y)$ is illustrated as a function of $y$ in Figure 1. As $y$ increases from 0 to $1, F(x, y)$ increases rapidly from 0 to infinity for all ranges of $x$. When $x>0.16, F(x, y)$ is independent of $x$, and $F(x, y)=1$ at $y=0.3345$. This value of $y$ is $9 \%$ larger than the exact value $R_{z} / D=0.30776$

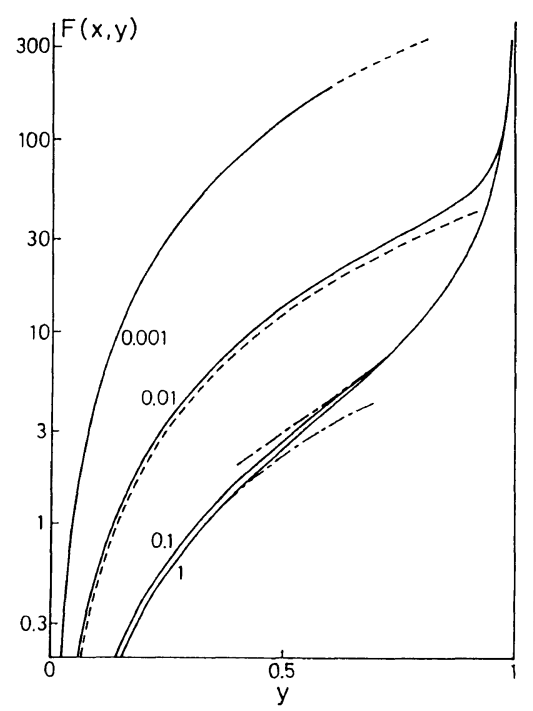

Figure 1. $F(x, y)$ as a function of $y$ at indicated $x$. The dotted curves are approximated values by eq $11 \mathrm{a}$; Dot-dash curves are approximated values by eq $11 \mathrm{~b}$ and $11 \mathrm{c}$. 
obtained from the mean end-to-end distance of an unperturbed chain in a thin slit. ${ }^{3}$ Equation 11a shows that $F\left(N / 6 D^{2}, R_{z} / D\right)$ equals $\alpha_{z}^{2}$ in the $D \rightarrow \infty$ limit. This means $\alpha_{x}=\alpha_{y}=\alpha_{z}=\alpha_{\infty}$ for an unconfined perturbed chain. Then we get from eq 7-9,

$$
\alpha_{\infty}^{5}-\alpha_{\infty}^{3}=c z
$$

This is the well-known Flory equation for the expansion factor of an unconfined chain. Substituting eq 12 into eq 7 (henceforth we set $\alpha_{x}=\alpha_{y}$, following the symmetry of the system), we get

$$
\left(\alpha_{x}^{4}-\alpha_{x}^{2}\right) \alpha_{z}=\alpha_{\infty}^{5}-\alpha_{\infty}^{3}
$$

Solving eq 13 for $\alpha_{z}$, and substituting it into eq 9 , we get

$$
F\left(\frac{R_{z \infty}^{2}}{2 D^{2} \alpha_{\infty}^{2}}, \frac{R_{z \infty}}{D} \frac{\left(\alpha_{\infty}^{4}-\alpha_{\infty}^{2}\right)}{\left(\alpha_{x}^{4}-\alpha_{x}^{2}\right)}\right)=\alpha_{x}^{2}
$$

using the relation $R_{z \infty}=(N / 3)^{1 / 2} \alpha_{\infty}$. Equation 14 can be solved numerically for $\alpha_{x}$ at given $D / R_{z \infty}$ and $\alpha_{\infty}^{2} . \alpha_{z}$ can be obtained using eq 13 .

The crossover concentration, $C^{*}$ is equal to the segment density of a polymer chain in a dilute solution, which is given as

$$
C^{*}=N / V \simeq N^{-1 / 2} / \alpha_{x}^{2} \alpha_{z}
$$

In $C<C^{*}$ (dilute regime) a chain behaves as a single chain, and chains penetrate each other in $C>C^{*}$ (semi-dilute regime). In an unconfined system, $C_{\infty}^{*}$ is given as

$$
C_{\infty}^{*} \sim N^{-1 / 2} \alpha_{\infty}^{-3}
$$

\section{Semi-Dilute Regime}

The parallel component of the expansion factor in a semi-dilute regime, $\alpha_{c x}=R_{c x} /(N / 3)^{1 / 2}$ is given using eq 5 in the same manner as eq 7 for a dilute regime. (Henceforth suffix $c$ denotes the value in a semi-dilute regime.)

$$
\alpha_{c x}^{2}-1=c^{\prime} z N^{-1 / 2} / \alpha_{c x}^{2} \alpha_{c y}^{2} \alpha_{c z}^{2} C
$$

where $c^{\prime}$ is a numerical constant. The following equations are also derived.

$$
\begin{gathered}
\alpha_{c y}^{2}-1=c^{\prime} z N^{-1 / 2} / \alpha_{c x}^{2} \alpha_{c y}^{2} \alpha_{c z}^{2} C \\
F\left(N / 6 D^{2}, R_{z} / D\right)-1=c^{\prime} z N^{-1 / 2} / \alpha_{c x}^{2} \alpha_{c y}^{2} \alpha_{c z}^{2} C
\end{gathered}
$$

Substituting eq 16 into eq 17 , we get

$$
\alpha_{c x}^{2}-1=c z \frac{\alpha_{\infty}^{3}}{\alpha_{c x}^{2} \alpha_{c y}^{2} \alpha_{c z}^{2}} \frac{C_{\infty}^{*}}{C}=\frac{\left(\alpha_{\infty}^{8}-\alpha_{\infty}^{6}\right)}{\alpha_{c x}^{4} \alpha_{c z}^{2}} \frac{C_{\infty}^{*}}{C}
$$

Here we set $\alpha_{c x}=\alpha_{c y}$, and eq 12 is used assuming that expansion factors are continuous for $C=C^{*}$. Equation 20 can be rewritten as

$$
\left(\alpha_{c x}^{6}-\alpha_{c x}^{4}\right) \alpha_{c z}^{2}=\left(\alpha_{\infty}^{8}-\alpha_{\infty}^{6}\right)\left(C_{\infty}^{*} / C\right)
$$

Solving eq 21 for $\alpha_{c z}$, and substituting it into eq 19 , we get

$$
F\left(\frac{R_{z \infty}^{2}}{2 D^{2} \alpha_{\infty}^{2}}, \frac{R_{z \infty}}{D}\left(\frac{\left(\alpha_{\infty}^{6}-\alpha_{\infty}^{4}\right)}{\left(\alpha_{c x}^{6}-\alpha_{c x}^{4}\right)} \frac{C_{\infty}^{*}}{C}\right)^{1 / 2}\right)=\alpha_{c x}^{2}
$$

Equation 22 can be solved numerically for $\alpha_{c x}$ at given $D / R_{z \infty}, C / C_{\infty}^{*}$ and $\alpha_{\infty}^{2}$.

\section{DISCUSSION}

\section{Dilute Regime}

The values of $\alpha_{x}^{2} / \alpha_{\infty}^{2}$ and $\alpha_{z}^{2} / \alpha_{\infty}^{2}$ at various $\alpha_{\infty}^{2}$ are plotted against $D / R_{z \infty}$ in Figure 2, and in Figure 3 on a double logarithmic scale. The dot-dash curve represents the exact $\alpha_{z}^{2} / \alpha_{\infty}^{2}$ for an unperturbed chain calculated using the Fourier transformation of eq 1 . This curve is close to within $10 \%$ the curve calculated for an unperturbed chain $\left(\alpha_{\infty}=1\right)$ using eq 13 and 14. The effect of confinement on the value of $\alpha_{z}^{2} / \alpha_{\infty}^{2}$ is remarkable for a wide range of $D / R_{z \infty}$. The values of $\alpha_{z}^{2} / \alpha_{\infty}^{2}$ decrease with decreasing $D / R_{z \infty}$, and $\alpha_{\infty}^{2}$. The values of $\alpha_{z}^{2} / \alpha_{\infty}^{2}$ decrease proportionally to $D^{2}$ at a small value of $D$.

Deviations of the values of $\alpha_{z}^{2}$ from $\alpha_{\infty}^{2}$ are smaller for large values of $\alpha_{\infty}^{2}$ than for small values of $\alpha_{\infty}^{2}$. These facts can be explained as follows. We get the following relation in the case of $D \ll R_{z \infty}$ using eq $11 \mathrm{c}$ and 14 .

$$
\alpha_{z} / \alpha_{\infty}=\left(D / R_{z \infty}\right) /\left(1+3 / \alpha_{x}^{2}\right)
$$

Equation 23 predicts that the values of $\alpha_{z}^{2} / \alpha_{\infty}^{2}$ at given $D / R_{z \infty}$ are large for large values of 


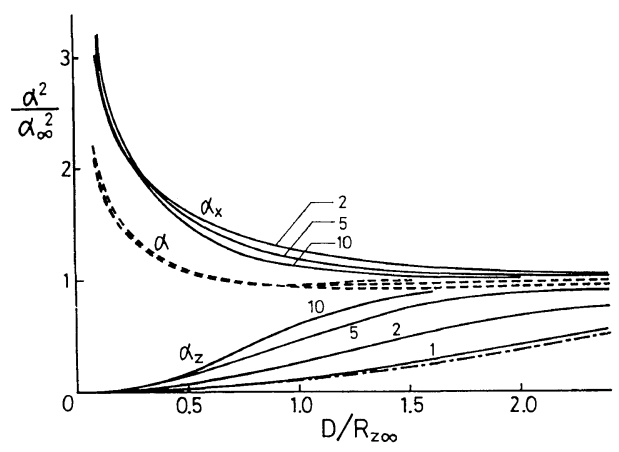

Figure 2. Reduced expansion factors as functions of $D / R_{z \infty}$. The symbols are denoted by $\alpha_{x}$, component parallel to the plates; $\alpha_{z}$, component perpendicular to the plates; $\alpha$, total expansion factor; Dot-dash curve, exact value for an unperturbed confined chain. The numbers in the figure indicate $\alpha_{\infty}^{2}$.

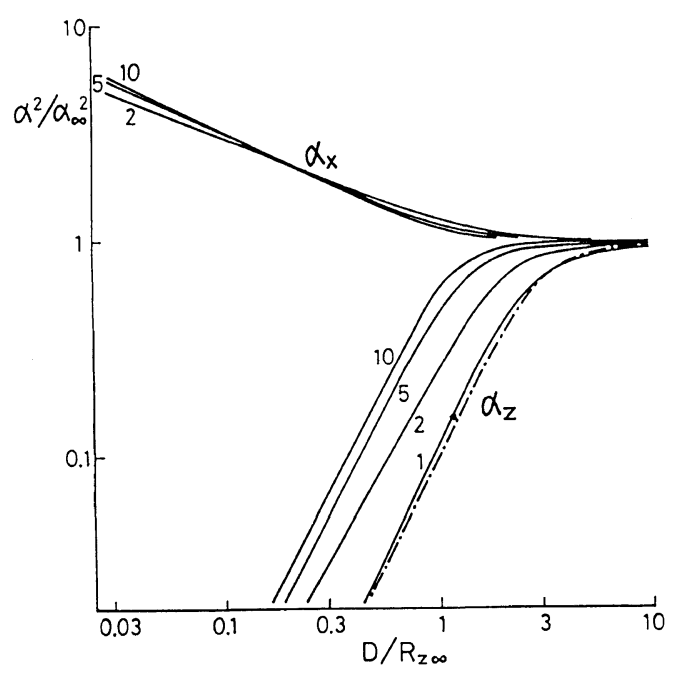

Figure 3. Double logarithmic plots of reduced expansion factors $v s . D / R_{z \infty}$. The symbols are the same as in Figure 2 .

$\alpha_{\infty}^{2}$, because the values of $\alpha_{x}^{2}$ are large in this case as discussed below.

The values of $\alpha_{x}^{2} / \alpha_{\infty}^{2}$ are more dependent on the values of $D / R_{z \infty}$ than on the value of $\alpha_{\infty}^{2}$. In the region $D \ll R_{z \infty}$, the values of $\alpha_{x}^{2} / \alpha_{\infty}^{2}$ for small values of $\alpha_{\infty}^{2}$ are larger than those for large values of $\alpha_{\infty}^{2}$. However, the values of $\alpha_{x}^{2} / \alpha_{\infty}^{2}$ for large values of $\alpha_{\infty}^{2}$ rapidly increase with decreasing $D / R_{z \infty}$, more so than those for small values of $\alpha_{\infty}^{2}$. Finally the former becomes larger than the latter, and increases proportion-

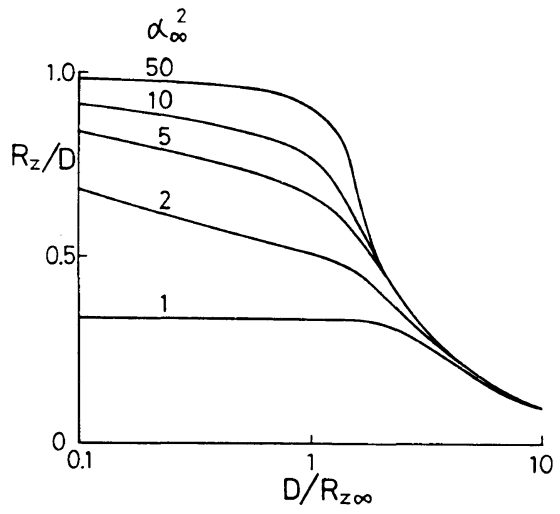

Figure 4. Component of the mean dimension perpendicular to the plates as a function of $D / R_{z \infty}$. The numbers in the figure indicate $\alpha_{\infty}^{2}$.

ally to $D^{-1 / 2}$.

The increasing value of $\alpha_{x}^{2} / \alpha_{\infty}^{2}$ with decreasing $D / R_{z \infty}$ is due to reduction of $R_{z}$ as explained by eq 7 . When a chain is compressed into $z$ direction, segments of the chain are squeezed in parallel directions to the plates owing to excluded volume interactions of segments.

The dotted curves in Figure 2 represent $\alpha^{2} / \alpha_{\infty}^{2}$. With decreasing $D / R_{z \infty}$, the value of $\alpha_{z}^{2} / \alpha_{\infty}^{2}$ decreases, and the value of $\alpha_{x}^{2} / \alpha_{\infty}^{2}$ increases monotonously. However, the value of $\alpha^{2} / \alpha_{\infty}^{2}$ takes a shallow minimum. This has been pointed out both by Monte Carlo simulations, ${ }^{12}$ and by our previous paper. ${ }^{3}$ It is noted that the values of $\alpha^{2} / \alpha_{\infty}^{2}$ are less dependent on the values of $\alpha_{\infty}^{2}$ than the values of $\alpha_{x}^{2} / \alpha_{\infty}^{2}$ and of $\alpha_{z}^{2} / \alpha_{\infty}^{2}$.

The values of $\alpha_{z}^{2} / \alpha_{\infty}^{2}$ rapidly approach 0 with decreasing $D$, because $R_{z}$ depends on $D$, and is independent of $N$ in the $D \rightarrow 0$ limit. In Figure 4 , the values of $R_{z} / D$ are plotted against $D / R_{z \infty}$. The value of $R_{z} / D$ is almost the same as the value of $R_{z \infty} / D$ while $D / R_{z \infty}>3$, regardless of the value of $\alpha_{\infty}^{2}$. The value of $R_{z} / D$ increases to 1 with decreasing $D / R_{z \infty}$, and increasing $\alpha_{\infty}^{2}$. The values of $R_{z} / D$ for an unperturbed chain stay 0.3345 for small values of $D / R_{z \infty}$ because $F\left(N / 6 D^{2}, R_{z} / D\right)=1$ in this case. 


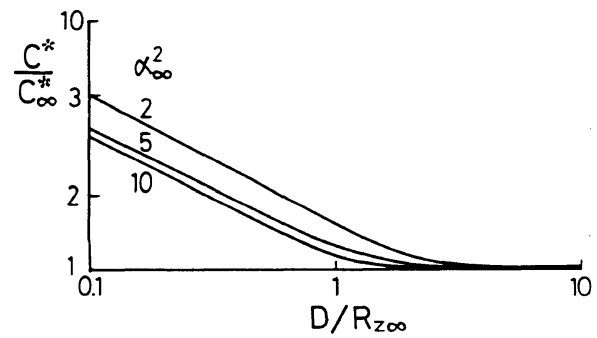

Figure 5. Reduced crossover concentration as a function of $D / R_{z \infty}$. The numbers in the figure indicate $\alpha_{\infty}^{2}$.

\section{Crossover Concentration}

The values of $C^{*} / C_{\infty}^{*}$ for indicated $\alpha_{\infty}^{2}$ are plotted against $D / R_{z \infty}$ in Figure 5. The values of $C^{*} / C_{\infty}^{*}$ increase with decreasing $D / R_{z \infty}$, and $\alpha_{\infty}^{2}$. Crossover from the dilute to the semi-dilute regime occurs at high concentrations with decreasing $D / R_{z \infty}$. This means that the segment density increases with decreasing $D / R_{z \infty}$. Slopes approach $-1 / 2$ in the $D / R_{z \infty} \rightarrow 0$ limit, because $R_{x}$ and $R_{z}$ are proportional to $D^{-1 / 4}$ and to $D$, respectively.

\section{Semi-Dilute Regime}

Values of $\alpha_{c x}^{2} / \alpha_{\infty}^{2}$ and $\alpha_{c z}^{2} / \alpha_{\infty}^{2}$ at indicated $C / C_{\infty}^{*}$ and $\alpha_{\infty}^{2}$ are plotted against $D / R_{z \infty}$ in Figure 6 . The dotted curves in the figure represent the values in a dilute regime. The values of $\alpha_{c x}^{2} / \alpha_{\infty}^{2}$ are more affected by the value of $D / R_{z \infty}$ than by the value of $\alpha_{\infty}^{2}$. The values of $\alpha_{c x}^{2} / \alpha_{\infty}^{2}$ are smaller than the value of $\alpha_{x}^{2} / \alpha_{\infty}^{2}$, and approach $\alpha_{x}^{2} / \alpha_{\infty}^{2}$ with decreasing $D / R_{z \infty}$. The values of $\alpha_{c x}^{2} / \alpha_{\infty}^{2}$ for small values of $C / C_{\infty}^{*}$ are larger than those for large values of $C / C_{\infty}^{*}$. The asymptotic values of $\alpha_{c x}^{2} / \alpha_{\infty}^{2}$ in the $D \rightarrow \infty$ limit decrease with increasing $C / C_{\infty}^{*}$.

The values of $\alpha_{c z}^{2} / \alpha_{\infty}^{2}$ decrease with decreasing $D / R_{z \infty}$, and approach those in the dilute regime, which decrease proportionally to $D^{2}$ for small value of $D / R_{z \infty}$. The values of $\alpha_{c z}^{2} / \alpha_{\infty}^{2}$ are more affected by the value of $\alpha_{\infty}^{2}$ than by the value of $C / C_{\infty}^{*}$ in the small $D / R_{z \infty}$ region.

In Figure 7, values of $\alpha_{c x}^{2} / \alpha_{\infty}^{2}$ are plotted against $C / C_{\infty}^{*}$ for indicated $D / R_{z \infty}$ and $\alpha_{\infty}^{2}$. The slopes are mainly dependent on the values of $\alpha_{\infty}^{2}$, and tend to $-1 / 4$ with increasing $\alpha_{\infty}^{2}$ and
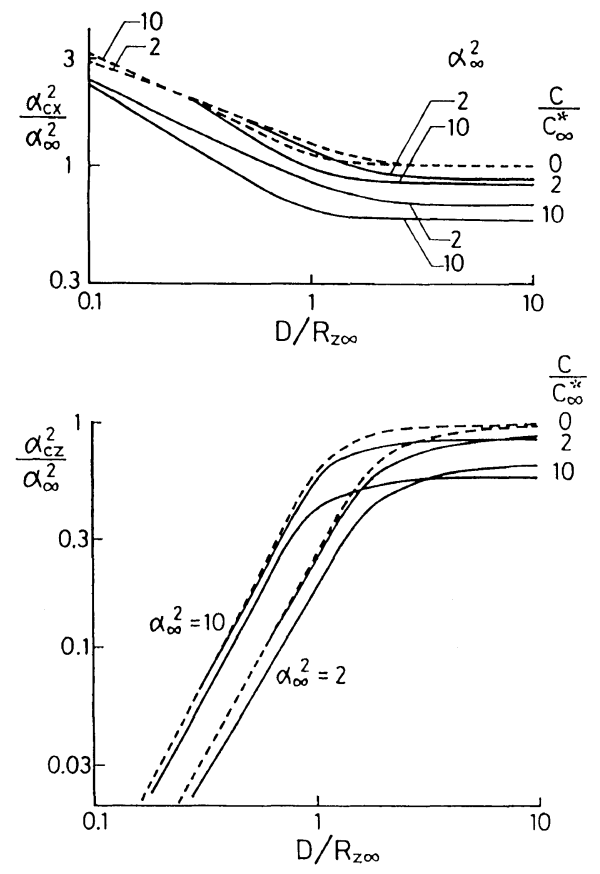

Figure 6. Reduced expansion factors as functions of $D / R_{z \infty}$. Top, component parallel to the plates; bottom, component perpendicular to the plates. Full curves, in a semi-dilute regime; dot curves, in a dilute regime. The numbers in the figure and on the right hand side indicate $\alpha_{\infty}^{2}$ and $C / C_{\infty}^{*}$, respectively.

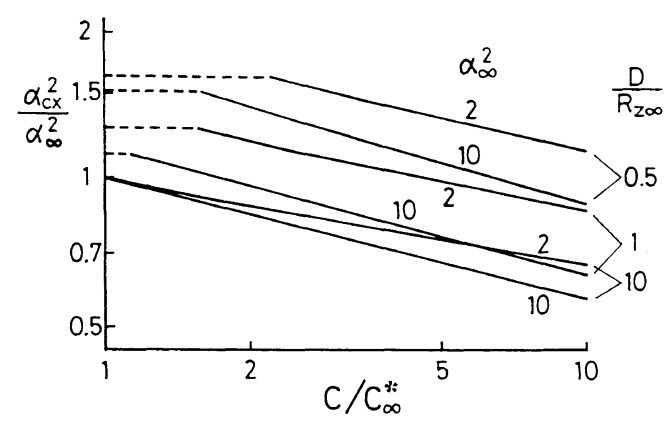

Figure 7. Component of reduced expansion factor parallel to the plates as a function of $C / C_{\infty}^{*}$. The numbers in the figure and on the right hand side indicate $\alpha_{\infty}^{2}$ and $D / R_{z \infty}$, respectively.

$D / R_{z \infty}$. The dotted curves represent the values in a dilute regime. The cross point of the full curve and the dotted curve corresponds to the crossover concentration.

In Figure 8 , the values of $R_{z} / D$ are plotted 


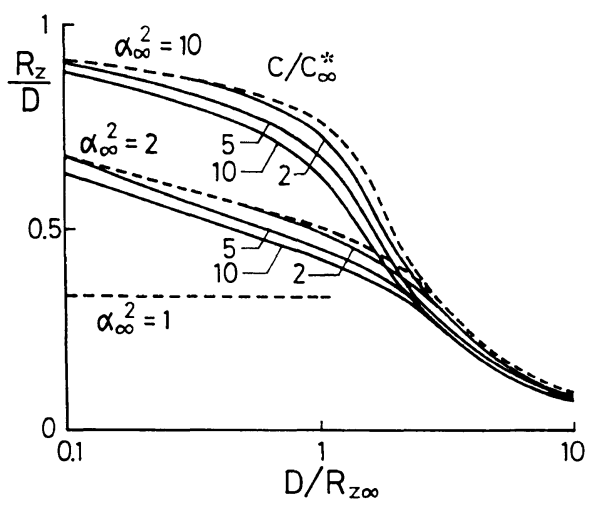

Figure 8. Component of the mean dimension perpendicular to the plates as a function of $D / R_{z \infty}$. The numbers in the figure indicate $\alpha_{\infty}^{2}$ and $C / C_{\infty}^{*}$.

against $D / R_{z \infty}$. The value of $R_{z} / D$ is similar to the value of $R_{z \infty} / D$ while $D / R_{z \infty}>3$, regardless the values of $C / C_{\infty}^{*}$ and $\alpha_{\infty}^{2}$. The values of $R_{z} / D$ increase to 1 with decreasing $D / R_{z \infty}$ and increasing $\alpha_{\infty}^{2}$, similarly to those in a dilute regime. The values of $R_{z} / D$ increase gradually with decreasing $C / C_{\infty}^{*}$.

\section{Limiting Cases}

The simple relation between $\alpha_{x}^{2}$ and $D$ in the $D / R_{z \infty} \rightarrow 0$ limit can be obtained easily as follows. We put $R_{z}=D$ in eq 4 , which gives us

$$
\alpha_{x}^{2}-1=c z(N / 3)^{1 / 2} / D \alpha_{x}^{2}
$$

instead of eq 7. Substituting eq 12 into eq 24 , the following relation can be derived.

$$
\alpha_{x}^{4}-\alpha_{x}^{2}=\left(\alpha_{\infty}^{4}-\alpha_{\infty}^{2}\right) R_{z \infty} / D
$$

If $\alpha_{\infty}^{2} \gg 1$, the terms of order $\alpha^{2}$ can be neglected. We have

$$
\alpha_{x}^{2} / \alpha_{\infty}^{2}=\left(R_{z \infty} / D\right)^{1 / 2}
$$

Equation 26 can be rewritten as

$$
R_{x}^{2} \sim N^{3 / 2} D^{-1 / 2}
$$

Equation 27 is consistent with the scaling prediction, ${ }^{1}$ and with the results by Monte Carlo simulations. ${ }^{13}$

The relation between $\alpha_{c x}^{2}$ and $D$ in the $D / R_{z \infty} \rightarrow 0$ limit can be obtained in the same

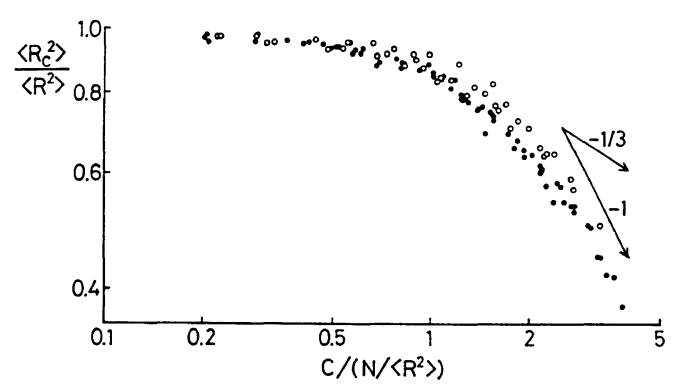

Figure 9. Reduced mean square end-to-end distance of self-avoiding chains in 2-dimensional lattice as a function of reduced concentration. ${ }^{14}$, triangular lattice; $\bigcirc$, square lattice.

manner. We get

$$
\alpha_{c x}^{2}-1 \sim z N^{1 / 2} / D^{2} \alpha_{c x}^{4} C
$$

instead of eq 17. Substituting eq 12 into eq 28 , we can derive

$$
\alpha_{c x}^{6}-\alpha_{c x}^{4}=\left(\alpha_{\infty}^{6}-\alpha_{\infty}^{4}\right)\left(\frac{R_{z \infty}}{D}\right)^{2} \frac{C_{\infty}^{*}}{C}
$$

If $\alpha_{\infty}^{2} \gg 1$, we have

$$
R_{c x}^{2} \sim N^{4 / 3} D^{-2 / 3} C^{-1 / 3}
$$

Equation 30 is not consistent with the scaling prediction. $^{1}$

Unfortunately, no experimental data of the chain dimensions of a confined chain in a semi-dilute regime are available at present. However Monte Carlo simulations on the concentration dependence of the dimensions of self-avoiding chains on two dimensional lattices were reported by Okamoto, Itoh and Takabumi. ${ }^{14}\left\langle R_{c}^{2}\right\rangle /\left\langle R^{2}\right\rangle$ of their data are plotted against $C /\left(N /\left\langle R^{2}\right\rangle\right)$ in Figure 9 . $N /\left\langle R^{2}\right\rangle$ is proportional to $C^{*}$ in two dimensional space. $\left\langle R_{c}^{2}\right\rangle /\left\langle R^{2}\right\rangle$ seems to be a universal function of $C /\left(N /\left\langle R^{2}\right\rangle\right)$. The exponent of concentration dependence seems to be close to -1 , which is more closely predicted by the scaling theory ${ }^{1}$ than the present value of $-1 / 3$. The reason for this difference is not clear at present, but it is noteworthy that the mean field theory gives the same exponent as predicted by the scaling theory in quasi one 


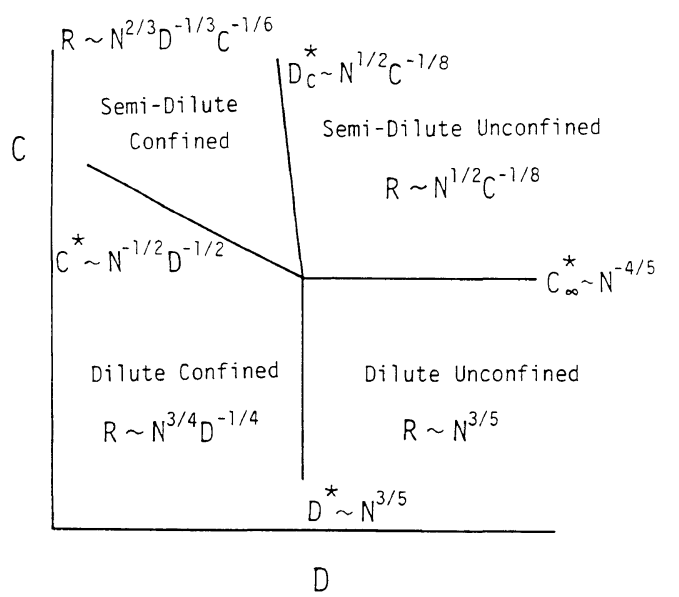

Figure 10. A sketch of the plate distance-concentration diagram of a polymer solution confined between two plates.

dimensional space as shown in the Appendix. In the $D \rightarrow \infty$ limit, we can put $\alpha_{c x}=\alpha_{c y}=\alpha_{c z}$ $\left(=\alpha_{c \infty}\right)$, then eq 21 can be rewritten as

$$
\alpha_{c \infty}^{8}-\alpha_{c \infty}^{6}=\left(\alpha_{\infty}^{8}-\alpha_{\infty}^{6}\right)\left(C_{\infty}^{*} / C\right)
$$

If $\alpha_{\infty}^{2} \gg 1$, eq 31 predicts

$$
R_{c \infty}^{2} \sim N C^{-1 / 4}
$$

Equation 32 is consistent with the scaling prediction by Daoud and Jannink. ${ }^{5}$

\section{Diagram for a Confined Polymer Solution}

In Figure 10, the plate distance-concentration diagram for a polymer solution confined between two plates is proposed. The diagram is composed of 4 different regimes. The chain dimensions in these regimes at the scaling limit are given as follows. In a dilute unconfined regime, the chain dimensions are evaluated using eq 12. The dimensions in a dilute confined regime are given by eq 25 or eq 27 . The crossover distance, $D^{*}$ is on the order of the dimensions of an unconfined chain. The dimensions in a semi-dilute unconfined regime can be obtained using eq 31 or eq 32 . The crossover concentration is equal to the segment density of an unconfined chain, and is given by eq 16 . In a semi-dilute confined regime, the dimensions can be predicted by eq 29 or eq 30 .
The crossover concentration increases with decreasing $D$ as predicted by eq 15 . The crossover distance is on the order of $R_{c \infty}$. It decreases with increasing $C$ as predicted by eq 32 .

\section{CONCLUSIONS}

The components of expansion factors perpendicular and parallel to the plates are derived as functions of $D / R_{z \infty}, \alpha_{\infty}^{2}$, and $C / C_{\infty}^{*}$ in a dilute and in a semi-dilute regime. In the limit of $D \rightarrow 0$, simple closed expressions, eq 25 and eq 29 are derived. The former is consistent with the scaling prediction, ${ }^{1}$ while the latter is inconsistent with it.

The diagram for a polymer solution confined between two plates is proposed. Chain dimensions at the scaling limit are given in each regime. The crossover lines are also given.

Acknowledgments. The author is grateful to Prof. H. Okamoto of Nagoya Institute of Technology for his provision of simulation data. This work was supported in part by a Grant-in-Aid for Scientific Research from the Ministry of Education, Science, and Culture of Japan.

\section{APPENDIX}

The chain dimensions of a polymer confined in quasi one dimensional space (for instance, in a capillary) were studied both by Daoud and deGennes, ${ }^{1}$ and by Turban. ${ }^{10}$ They used the relation, $R_{x} \sim N$ to determine the exponent in the scaling function of two length scales, $R_{x}$ and $D$ in a dilute regime. However, the exponent on concentration in the scaling function for a semi-dilute regime could not be directly determined. They used the blob model to determine it.

Here we derived a relation between the expansion factor and the diameter of a capillary, $D$ in the limit of $D \rightarrow 0$.

If we put $R_{y}=R_{z}=D$ into eq 6 , we get 


$$
\alpha_{x}^{2}-1=c z(N / 3) / D^{2} \alpha_{x}
$$

instead of eq 24 . Substituting eq 12 into eq $A 1$, the following relation is derived.

$$
\alpha_{x}^{3}-\alpha_{x}=\left(\alpha_{\infty}^{3}-\alpha_{\infty}\right)\left(R_{z \infty} / D\right)^{2}
$$

If $\alpha_{\infty}^{2} \gg 1$, we have,

$$
R_{x} \sim N D^{-2 / 3}
$$

Equation $\mathrm{A} 3$ is consistent with the scaling prediction. ${ }^{1,10}$

In the same manner, we get

$$
\alpha_{c x}^{2}-1 \sim z N^{3 / 2} / D^{4} \alpha_{c x}^{2} C
$$

instead of eq 28. Substituting eq 12 into eq A4, we have

$$
\alpha_{c x}^{4}-\alpha_{c x}^{2}=\left(\alpha_{\infty}^{4}-\alpha_{\infty}^{2}\right)\left(\frac{R_{z \infty}}{D}\right)^{4} \frac{C_{\infty}^{*}}{C}
$$

If $\alpha_{\infty}^{2} \gg 1$, eq A5 can be rewritten as

$$
R_{c x} \sim N D^{-1} C^{-1 / 4}
$$

Equation A6 is also consistent with the scaling prediction. ${ }^{1,10}$

\section{REFERENCES}

1. M. Daoud and P. G. deGennes, J. Physique, 38, 85 (1977).

2. Z. Wang, A. M. Nemirovsky, and K. F. Freed, J. Chem. Phys., 86, 4266 (1987).

3. K. Shiokawa, Polym. J., 22, 925 (1990).

4. Y. Oono, Phys. Rev., A30, 986 (1984).

5. M. Daoud and G. Jannink, J. Physique, 37, 973 (1976).

6. S. F. Edwards and E. F. Jeffers, J. Chem. Soc., Faraday Trans. II, 75, 1020 (1979).

7. T. Oyama and K. Shiokawa, Polym. J., 12, 507 (1980).

8. T. Ohta and Y. Oono, Phys. Lett., 89A, 460 (1982).

9. M. Fisher, J. Phys. Soc. Jpn., 26 (Suppl.), 44 (1969).

10. L. Turban, J. Physique, 45, 347 (1984).

11. J. J. Hermans and J. T. G. Overbeek, Rec. Trav. Chim., 67, 761 (1948).

12. F. T. Wall, F. Mandel, and J. C. Chin, J. Chem. Phys., 65, 2231 (1976).

13. For example, I. Webman, J. L. Lebowitz, and M. H. Kalos, J. Physique, 41, 579 (1980).

14. H. Okamoto, K. Itoh, and A. Takabumi, J. Chem. Phys., 78, 975 (1983). 